\title{
Lente de contato de material híbrido em pacientes com ceratocone e astiǵmatismo miópico composto
}

\author{
Hybrid material contact lens in keratoconus and myopic astigmatism patients
}

\author{
Fernando Leal ${ }^{1}$ \\ César Lipener ${ }^{2}$ \\ Maria Regina Chalita ${ }^{3}$ \\ Ricardo Uras ${ }^{4}$ \\ Mauro Campos 5 \\ Ana Luisa Höfling'-Lima ${ }^{6}$
}

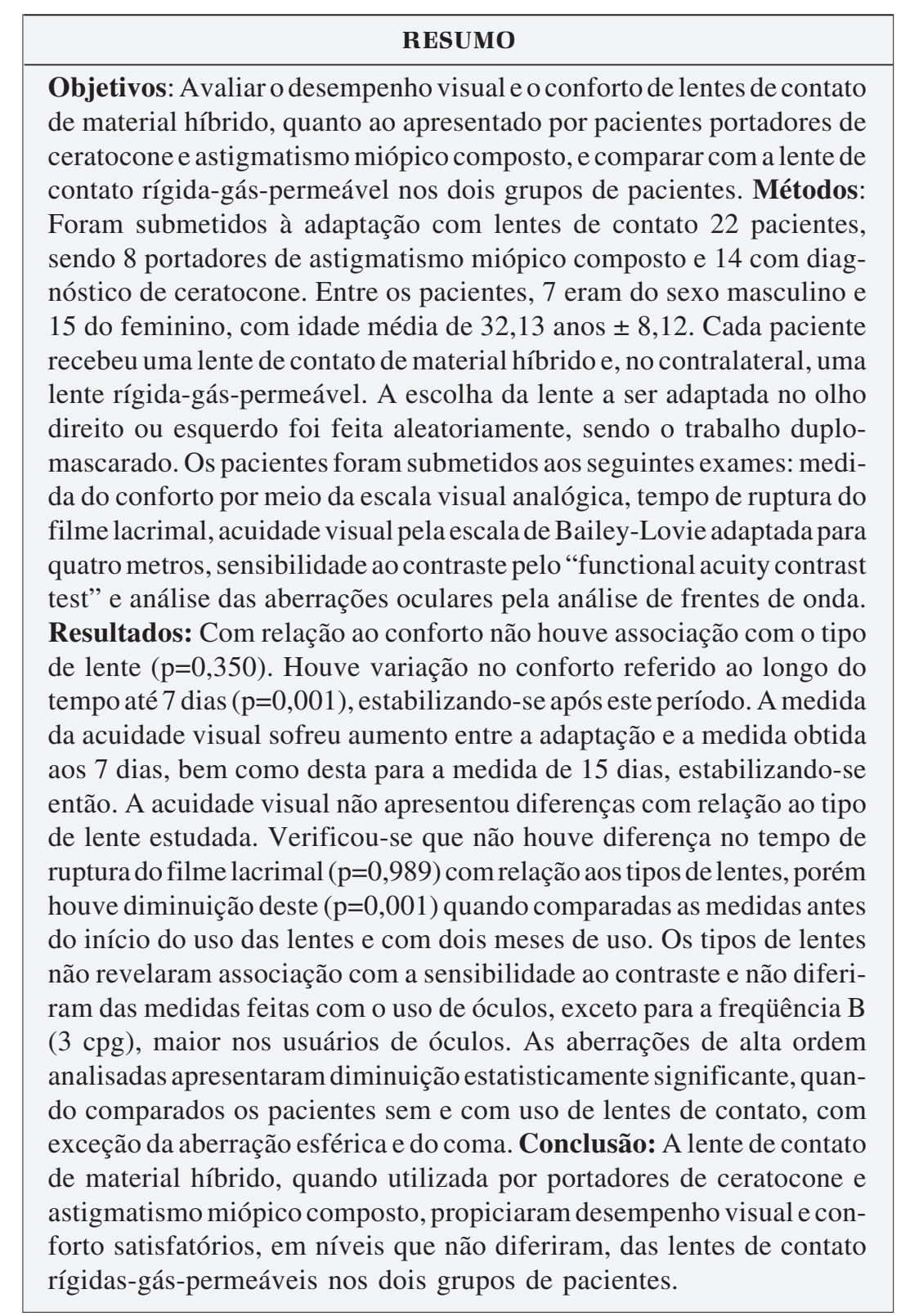

Descritores: Lentes de contato; Ceratocone; Miopia; Astigmatismo; Sensibilidade de contraste

Objetivos: Avaliar o desempenho visual e o conforto de lentes de contato de material híbrido, quanto ao apresentado por pacientes portadores de ceratocone e astigmatismo miópico composto, e comparar com a lente de contato rígida-gás-permeável nos dois grupos de pacientes. Métodos: Foram submetidos à adaptação com lentes de contato 22 pacientes, sendo 8 portadores de astigmatismo miópico composto e 14 com diagnóstico de ceratocone. Entre os pacientes, 7 eram do sexo masculino e 15 do feminino, com idade média de 32,13 anos $\pm 8,12$. Cada paciente lente rígida-gás-permeável. A escolha da lente a ser adaptada no olho direito ou esquerdo foi feita aleatoriamente, sendo o trabalho duploda do conforto por meio da escala visual analógica, tempo de ruptura do filme lacrimal, acuidade visual pela escala de Bailey-Lovie adaptada para Resultados: Com relação ao conforto não houve associação com o tipo de lente $(\mathrm{p}=0,350)$. Houve variação no conforto referido ao longo do tempo até 7 dias ( $\mathrm{p}=0,001)$, estabilizando-se após este período. A medida da acuidade visual sofreu aumento entre a adaptação e a medida obtida aos 7 dias, bem como desta para a medida de 15 dias, estabilizando-se então. A acuidade visual não apresentou diferenças com relação ao tipo de lente estudada. Verificou-se que não houve diferença no tempo de houve diminuição deste $(\mathrm{p}=0,001)$ quando comparadas as medidas antes do início do uso das lentes e com dois meses de uso. Os tipos de lentes

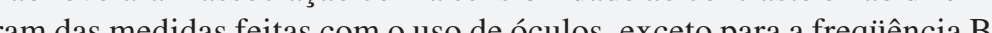
(3 cpg), maior nos usuários de óculos. As aberrações de alta ordem analisadas apresentaram diminuição estatisticamente significante, quando comparados os pacientes sem e com uso de lentes de contato, com exceção da aberração esférica e do coma. Conclusão: A lente de contato de material híbrido, quando utilizada por portadores de ceratocone e astigmatismo miópico composto, propiciaram desempenho visual e conforto satisfatórios, em níveis que não diferiram, das lentes de contato rígidas-gás-permeáveis nos dois grupos de pacientes.

\footnotetext{
Trabalho realizado no setor de Lentes de Contato do Departamento de Oftalmologia da Escola Paulista de Medicina/Universidade Federal de São Paulo.

${ }^{1}$ Doutorando em oftalmologia pela Universidade Federal de São Paulo - UNIFESP - São Paulo (SP) - Brasil.

${ }^{2}$ Mestre em Oftalmologia e chefe do setor de Lentes de Contato pela UNIFESP - São Paulo (SP) - Brasil.

${ }^{3}$ Doutora em Oftalmologia pela UNIFESP - São Paulo (SP) - Brasil.

${ }^{4}$ Doutor em Oftalmologia e Diretor do Instituto da Visão pela UNIFESP - São Paulo (SP) - Brasil.

${ }^{5}$ Livre Docente em oftalmologia e chefe do Setor de Cirurgia Refrativa pela UNIFESP - São Paulo (SP) Brasil.

${ }^{6}$ Professora Titular de Oftalmologia e chefe do Departamento de Oftalmologia da UNIFESP - São Paulo (SP) Brasil

Endereço para correspondência: Fernando Leal. Rua Vereador Edmundo Genoino de Oliveira, 2954/201 Teresina (PI) CEP 64055-030

E-mail:faml@terra.com.br

Recebido para publicação em 08.05.2006

Última versão recebida em 23.08.2006

Aprovação em 27.09.2006

Este trabalho foi realizado com auxilio financeiro da Coordenação de Aperfeiçoamento de Pessoal de Nível Superior (CAPES).
} 


\section{INTRODUÇÃO}

A história do desenvolvimento das lentes de contato (LCs) está intimamente relacionada com a idéia de se alcançar, ao mesmo tempo, melhor acuidade visual e máximo conforto, aliadas ao menor número de complicações possíveis. Partindo deste princípio, a invenção de novos materiais tem influenciado o aperfeiçoamento de novas lentes de contato ${ }^{(1)}$.

Desde a descoberta das LCs em 1888, uma evolução constante tem levado à utilização de novos materiais e produção de lentes cada vez mais confortáveis, propiciando melhor desempenho visual, ampliando o tempo de uso e, conseqüentemente, aumentando as possibilidades de uma boa adaptação ${ }^{(2-3)}$.

Esta evolução levou à produção de dois grandes grupos de lentes, que do ponto de vista físico, gerou a classificação destas como rígidas e gelatinosas. Atualmente, a indústria tem se concentrado no desenvolvimento de lentes que sejam capazes de reunir as melhores propriedades dos materiais rígidos permeáveis aos gases e dos gelatinosos, também chamados hidrofílicos ${ }^{(3-4)}$.

As primeiras pesquisas no desenvolvimento dos materiais gelatinosos foram realizadas em 1971 com lentes que continham um material hidrofílico formado a partir do hidroxietilmetacrilato (HEMA), material gelatinoso, e do polimetilmetacrilato (PMMA), material rígido não permeável aos gases. Com o surgimento dos materiais rígidos-gás-permeáveis (RGPs), desenvolveram-se lentes cujo centro era formado a partir deste material circundado por uma banda hidrofílica. Não era um novo material e sim a utilização de uma lente de contato que continha materiais em estados físicos diferentes. Tais lentes foram chamadas de lentes de contato híbridas ${ }^{(5)}$.

As investigações nesse sentido levaram ao desenvolvimento dos polímeros flexíveis. Esta tecnologia de síntese polimérica criou uma estrutura molecular que se diferencia das lentes gelatinosas tradicionais e das clássicas lentes rígidasgás-permeáveis. São, portanto, um novo material que tem como objetivo combinar as qualidades ópticas do material rígido-gás-permeável (RGP) com as qualidades do material gelatinoso que denominamos lentes de contato de material híbrido $(\mathrm{MH})^{(6)}$.

\section{OBJETIVOS}

Este estudo teve como objetivos: avaliar o desempenho visual e o conforto de lentes de contato de material híbrido, quanto ao apresentado por pacientes portadores de ceratocone e astigmatismo miópico composto, e comparar com a lente de contato rígida-gás-permeável nos dois grupos de pacientes.

\section{MÉTODOS}

Foram submetidos à adaptação com lentes de contato 22 pacientes, sendo oito portadores de astigmatismo miópico composto e 14 com diagnóstico topográfico de ceratocone, utilizando-se para tal os mapas paquimétricos e ceratométricos do Orbscan ${ }^{\circledR}$ II (Baush e Lomb, Inc., Nova Iorque, EUA). Entre os pacientes, 7 eram do sexo masculino e 15 do feminino, com idade média de 32,13 anos $\pm 8,12$ (Quadro 1). Cada paciente recebeu em um olho uma lente de contato de material híbrido e, no contralateral, uma lente rígida-gás-permeável (Solótica ${ }^{\circledR}$ DKs 23 e 30, respectivamente). A escolha da lente a ser adaptada no olho direito ou esquerdo foi feita aleatoriamente, sendo o trabalho duplo-mascarado. Os seguintes critérios de inclusão foram adotados:

- Pacientes ainda não usuários e usuários de LC e sem história de intolerância a estas.

- Pacientes que receberam correção com lentes de contato bilateralmente.

- Pacientes que alcancem visão superior ou igual a 0,5 (tabela de Bailey-Lovie para 4 metros) com melhor correção.

- Pacientes portadores de astigmatismo miópico composto em ambos os olhos.

- Pacientes com componente miópico menor ou igual a 10 dioptrias esféricas.

- Pacientes portadores de astigmatismo menor ou igual a cinco dioptrias cilíndricas.

- Pacientes cuja diferença no grau esférico em ambos os olhos seja menor ou igual a três dioptrias.

- Pacientes cuja diferença no grau cilíndrico em ambos os olhos seja menor ou igual a três dioptrias.

Os pacientes foram submetidos ao exame oftalmológico incluindo: acuidade visual com correção através da escala de Bailey-Lovie para 4 metros, esquiascopia, biomicroscopia, oftalmoscopia indireta, ceratometria e topografia corneana. Os mesmos foram submetidos à adaptação em um olho com lente rígida-gás-permeável e no olho contralateral com lente de material híbrido. Para cada olho foram feitos dois testes: um com lente RGP e outro com lente de material híbrido. A escolha da lente a ser adaptada em cada olho foi feita aleatoriamente.

As lentes foram entregues aos pacientes após os mesmos serem informados e treinados sobre a colocação, remoção e manutenção das mesmas. Os pacientes foram orientados a usar a solução multiuso Opti-Free Express ${ }^{\circledR}$ e Supraclens ${ }^{\circledR}$ para desproteinização. A lente direita foi marcada com um sinal em forma de linha reta, para que não ocorressem trocas das mesmas. Foi anotado, pelo examinador o número de horas por dia, para cada olho separadamente, que cada paciente fez uso das lentes de contato.

O conforto experimentado pelo paciente em cada olho foi avaliado separadamente através da escala visual analógica adaptada para conforto, no qual os parâmetros extremos foram: ausência de desconforto e pior desconforto imaginável (Figura 1). Esta análise foi feita no momento e 10 minutos após o início do uso das lentes e nos $7^{\circ}, 15^{\circ}, 30^{\circ}$ e $60^{\circ}$ dias subsequentes. As medidas do tempo de ruptura do filme lacrimal foram obtidas antes da adaptação e com 2 meses após o início do uso das lentes de contato.

A medida da acuidade visual foi aferida com a escala de Bailey-Lovie adaptada para a distância de 4 metros no momen- 


\begin{tabular}{|c|c|c|c|c|c|c|c|}
\hline \multirow[b]{2}{*}{ Pacientes } & \multirow[b]{2}{*}{ Sexo } & \multirow[b]{2}{*}{ Idade } & \multicolumn{4}{|c|}{ Quadro 1. Dados gerais dos pacientes incluídos neste estudo } & \multirow[b]{2}{*}{ Tipo de lente } \\
\hline & & & Olho & Equivalente esférico & Ceratometria & Diagnóstico & \\
\hline \multirow[t]{2}{*}{1} & $\mathrm{~F}$ & 21 & OD & $-2,25$ & $45,6 \times 42,4$ & Ceratocone & $\mathrm{MH}$ \\
\hline & & & OE & $-2,00$ & $45,8 \times 42,4$ & Ceratocone & RGP \\
\hline \multirow[t]{2}{*}{2} & $\mathrm{~F}$ & 41 & OD & $-6,50$ & $45,9 \times 42,7$ & Ceratocone & $\mathrm{MH}$ \\
\hline & & & OE & $-6,75$ & $45,7 \times 42,8$ & Ceratocone & RGP \\
\hline \multirow[t]{2}{*}{3} & $\mathrm{~F}$ & 52 & OD & $-2,50$ & $42,9 \times 41,7$ & AMC & RGP \\
\hline & & & OE & $-4,00$ & $43,5 \times 42,0$ & AMC & $\mathrm{MH}$ \\
\hline \multirow[t]{2}{*}{$4^{*}$} & $M$ & 42 & OD & $-5,75$ & $45,5 \times 44,3$ & Ceratocone & RGP \\
\hline & & & OE & $-5,75$ & $47,1 \times 44,9$ & Ceratocone & $\mathrm{MH}$ \\
\hline \multirow[t]{2}{*}{5} & $M$ & 30 & OD & $-5,25$ & $45,7 \times 43,4$ & Ceratocone & $\mathrm{MH}$ \\
\hline & & & OE & $-5,50$ & $45,8 \times 43,5$ & Ceratocone & RGP \\
\hline \multirow[t]{2}{*}{6} & $F$ & 27 & OD & $-4,00$ & $47,1 \times 44,1$ & Ceratocone & $\mathrm{MH}$ \\
\hline & & & OE & $-3,00$ & $46,9 \times 44,0$ & Ceratocone & RGP \\
\hline \multirow[t]{2}{*}{7} & $\mathrm{~F}$ & 31 & OD & $-4,00$ & $44,1 \times 42,5$ & Ceratocone & $\mathrm{MH}$ \\
\hline & & & OE & $-5,50$ & $44,7 \times 43,5$ & Ceratocone & RGP \\
\hline \multirow[t]{2}{*}{$8^{*}$} & $M$ & 27 & OD & $-3,25$ & $44,1 \times 41,5$ & Ceratocone & $\mathrm{MH}$ \\
\hline & & & OE & $-4,75$ & $44,6 \times 41,3$ & Ceratocone & RGP \\
\hline \multirow[t]{2}{*}{9} & $\mathrm{~F}$ & 35 & OD & $-6,00$ & $47,5 \times 43,5$ & Ceratocone & RGP \\
\hline & & & OE & $-6,75$ & $47,2 \times 42,7$ & Ceratocone & $\mathrm{MH}$ \\
\hline \multirow[t]{2}{*}{10} & M & 35 & OD & $-2,75$ & $45,0 \times 42,8$ & Ceratocone & RGP \\
\hline & & & OE & $-2,00$ & $44,3 \times 43,0$ & Ceratocone & $\mathrm{MH}$ \\
\hline \multirow[t]{2}{*}{11} & $\mathrm{~F}$ & 28 & OD & $-9,50$ & $46,1 \times 43,0$ & AMC & RGP \\
\hline & & & OE & $-10,00$ & $46,6 \times 42,7$ & AMC & $\mathrm{MH}$ \\
\hline \multirow[t]{2}{*}{12} & $\mathrm{~F}$ & 36 & OD & $-2,75$ & $47,6 \times 45,7$ & Ceratocone & $\mathrm{MH}$ \\
\hline & & & OE & $-5,00$ & $48,9 \times 46,4$ & Ceratocone & RGP \\
\hline \multirow[t]{2}{*}{$13^{*}$} & $\mathrm{~F}$ & 35 & OD & $-2,00$ & $45,7 \times 44,2$ & Ceratocone & $\mathrm{MH}$ \\
\hline & & & OE & $-2,50$ & $48,6 \times 45,0$ & Ceratocone & RGP \\
\hline \multirow[t]{2}{*}{14} & $\mathrm{~F}$ & 24 & OD & $-7,00$ & $47,1 \times 43,4$ & Ceratocone & RGP \\
\hline & & & OE & $-6,50$ & $47,1 \times 43,7$ & Ceratocone & $\mathrm{MH}$ \\
\hline \multirow[t]{2}{*}{15} & M & 29 & OD & $-2,00$ & $44,5 \times 44,3$ & $\mathrm{AMC}$ & $\mathrm{MH}$ \\
\hline & & & OE & $-2,25$ & $44,9 \times 44,5$ & AMC & RGP \\
\hline \multirow[t]{2}{*}{16} & $\mathrm{~F}$ & 33 & OD & $-2,50$ & $44,4 \times 42,7$ & AMC & RGP \\
\hline & & & OE & $-4,50$ & $45,3 \times 43,0$ & AMC & $\mathrm{MH}$ \\
\hline \multirow[t]{2}{*}{17} & $\mathrm{~F}$ & 23 & OD & $-5,00$ & $46,0 \times 45,3$ & Ceratocone & RGP \\
\hline & & & OE & $-5,25$ & $46,1 \times 44,4$ & Ceratocone & $\mathrm{MH}$ \\
\hline 18 & M & 45 & OD & $-5,25$ & $43,7 \times 42,0$ & $\mathrm{AMC}$ & RGP \\
\hline & & & OE & $-5,25$ & $43,8 \times 42,3$ & AMC & $\mathrm{MH}$ \\
\hline 19 & $M$ & 23 & OD & $-4,75$ & $46,9 \times 46,1$ & AMC & RGP \\
\hline & & & OE & $-3,00$ & $46,2 \times 45,4$ & AMC & $\mathrm{MH}$ \\
\hline 20 & $\mathrm{~F}$ & 32 & OD & $-7,50$ & $45,8 \times 45,1$ & AMC & RGP \\
\hline & & & OE & $-7,75$ & $45,3 \times 44,2$ & AMC & $\mathrm{MH}$ \\
\hline 21 & $\mathrm{~F}$ & 22 & OD & $-2,50$ & $45,2 \times 43,8$ & Ceratocone & $\mathrm{MH}$ \\
\hline & & & OE & $-3,75$ & $45,1 \times 44,0$ & Ceratocone & RGP \\
\hline 22 & $\mathrm{~F}$ & 26 & OD & $-2,25$ & $46,7 \times 45,0$ & AMC & $\mathrm{MH}$ \\
\hline & & & OE & $-2,25$ & $46,2 \times 44,8$ & AMC & RGP \\
\hline
\end{tabular}

to de início do uso das lentes e no $7^{\circ}, 15^{\circ}, 30^{\circ}$ e $60^{\circ}$ dia após. A medida de sensibilidade ao contraste pelo método "functional acuity contrast test ${ }^{\circledR}($ FACT)" foi realizado com a melhor correção do paciente com o uso de óculos ou armação de prova e com o uso das lentes de contato.

Os pacientes foram submetidos à análise das aberrações oculares com o aberrômetro LADARWave ${ }^{\circledR}$ (AlconLabs, Fort Worth, TX) sem lentes e com as lentes de contato adaptadas. Foram analisadas as seguintes aberrações de baixa ordem: defocus, astigmatismo e seu eixo. Em relação às aberrações de alta ordem foram analisadas: coma, aberração esférica e aberrações de alta ordem. As aberrações totais (de baixa e alta ordens) também foram analisadas.

Devido ao fato de que os dados foram coletados sempre nos mesmos pacientes nas diferentes situações experimentais, empregou-se o modelo de análise de variância em blocos para o estudo estatístico e das associações de interesse. A análise das aberrações oculares foi realizada com a aplicação 

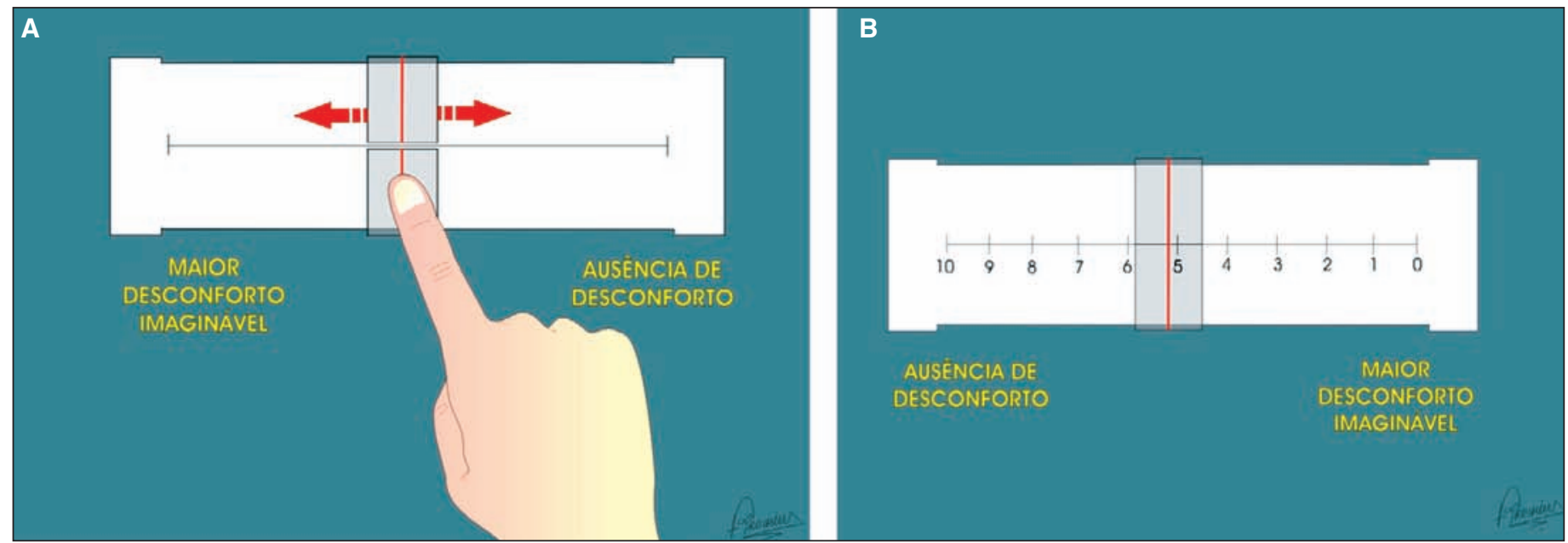

Figura 1 - A: Escala visual analógica - Frente. B: Escala visual analógica - Verso.

do método das comparações múltiplas de Bonferroni ${ }^{(7)}$, sendo que análise do astigmatismo e seu eixo foi realizada por meio de vetores conforme o método descrito por Naeser ${ }^{(8)}$.

\section{RESULTADOS}

Não se verificou associação do número de horas de uso com o tipo de lente $(\mathrm{p}=0,696)$, com os grupos de pacientes ( $\mathrm{p}=0,124)$ e com as medidas obtidas com 36 dias de uso das lentes $(\mathrm{p}=0,938)$.

Com relação à acuidade visual com correção, as medidas descritivas mostraram que a acuidade visual obtida com lentes de contato de material híbrido foi maior que a medida feita com óculos, independentemente do grupo de pacientes. As medidas obtidas com lente de material híbrido não foram diferentes estatisticamente das medidas feitas com lentes RGP, nos dois grupos de pacientes (Tabela 1, Figura 2).

Os resultados obtidos permitem afirmar que não houve associação da sensibilidade ao contraste com o tipo de lente e o grupo de pacientes estudados. Verificou-se, no entanto, que a sensibilidade aumentou quando se comparou a medida desta obtida com lente de MH com o uso de óculos para a frequiência B. As correções obtidas com ambas as lentes foram iguais para os grupos de pacientes nas freqüências A e B, porém foram diferentes quando se comparou, entre os grupos, as medidas nas freqüências C, D e E (Tabela 2, Figura 3).

Os níveis descritivos das comparações das aberrações oculares com os pacientes sem correção e com lentes de contato, de acordo com o grupo de pacientes e o tipo de lente de contato adaptada estão representados na tabela 3 .

As medidas das aberrações do componente esférico, cilíndrico e seu eixo, defocus, outras aberrações de alta ordem, aberração total (RMS) e aberrações de alta ordem (RMS) foram reduzidas com significância quando comparados os olhos adaptados com lentes com as medidas obtidas sem o uso destas. Nesta mesma comparação, as medidas da aberração

$\begin{aligned} & \text { Tabela 1. Resultados do estudo de associação entre acuidade } \\
& \text { visual obtida com lente de contato de material híbrido comparada } \\
& \text { com correção com óculos, lentes de contato rígida-gás-permeável } \\
& \text { e entre o grupo de pacientes }\end{aligned}$
\begin{tabular}{lc} 
Efeito & Nível descritivo \\
Óculos & 0,001 \\
Lente rígida-gás-permeável & 0,083 \\
Grupo de pacientes & 0,186 \\
\hline
\end{tabular}

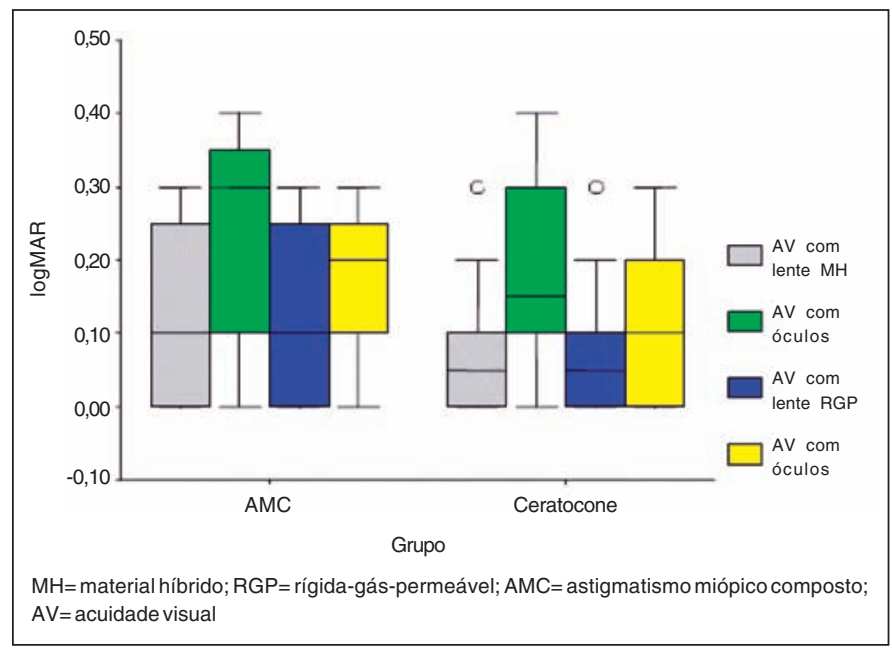

Figura 2 - Distribuição da acuidade visual segundo o tipo de correção óptica e o grupo de pacientes

Tabela 2. Níveis descritivos da comparação entre os tipos de lente e os tipos de correção para cada freqüência do exame de sensibilidade ao contraste

\begin{tabular}{lccccc} 
Freqüência & A & B & C & D & E \\
Tipo de lente & 0,641 & 0,724 & 0,568 & 0,755 & 0,293 \\
Tipo de correção & 0,125 & 0,019 & 0,451 & 0,368 & 0,151 \\
Grupo de pacientes & 0,798 & 0,239 & 0,021 & 0,026 & 0,006 \\
A=1,5 cpg; B=3 cpg; C= 6 cpg; $D=12 \mathrm{cpg} ; \mathrm{E}=18 \mathrm{cpg}$ & & \\
\hline
\end{tabular}


esférica e do coma, aumentaram e diminuíram, respectivamente; porém sem significância. Com relação ao tipo de lente estudada, não houve diferença significante em nenhuma das variáveis estudadas, no grupo total de pacientes e nos subgrupos. No entanto, houve diferença significante quando se comparou as variáveis: outras aberrações de alta ordem e as aberrações de alta ordem (RMS), entre os grupos de pacientes, para os dois tipos de lentes estudadas (Figuras 4 a 8).

Com relação ao conforto verificou-se que: o tipo de lente não apresentou associação com o conforto referido pelos pacientes $(\mathrm{p}=0,350)$ e que não houve diferença entre o grupo de pacientes com astigmatismo miópico composto e nos com ceratocone $(\mathrm{p}=0,909)$. Houve variação deste ao longo do tempo $(\mathrm{p}=0,001)$ com aumento na avaliação até a medida tomada com 7 dias, a partir de então, o conforto estabilizou-se (Figuras 9 e 10).

Verificou-se que não houve diferença entre as lentes de material híbrido e as rígidas gás-permeáveis ao longo do tem-

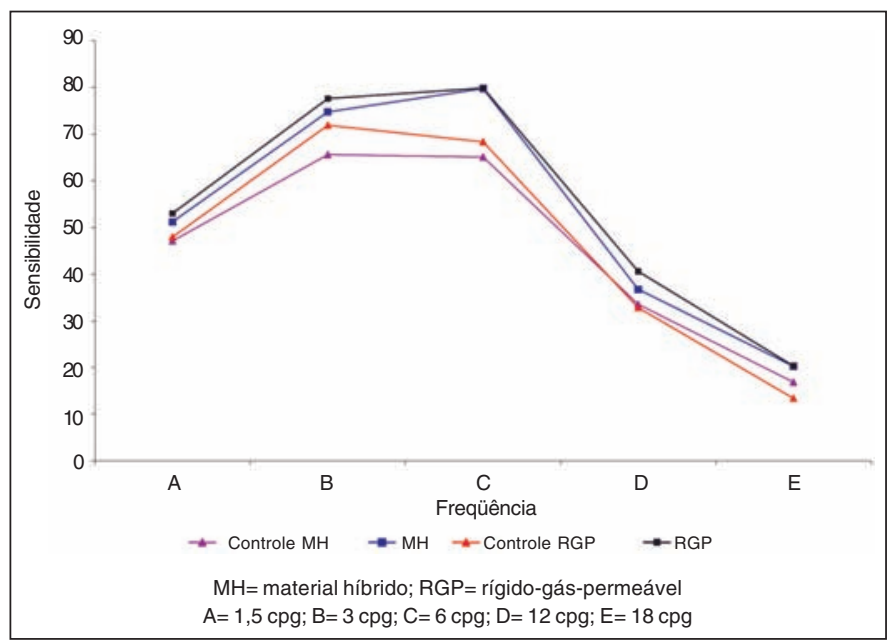

Figura 3 - Médias de sensibilidade ao contraste segundo o tipo de lente de contato e os controles usuários de óculos

\begin{tabular}{|c|c|c|c|}
\hline Variável & $\begin{array}{l}\text { Tempo com } \\
\text { e sem lente }\end{array}$ & $\begin{array}{l}\text { Tipo de lente } \\
\text { adaptada }\end{array}$ & $\begin{array}{l}\text { Grupo de } \\
\text { pacientes }\end{array}$ \\
\hline Esférico & 0,001 & 0,999 & 0,935 \\
\hline $\begin{array}{l}\text { Análise vetorial } \\
\text { do cilindro* }\end{array}$ & 0,001 & 0,999 & 0,140 \\
\hline Defocus & 0,001 & 0,999 & 0,678 \\
\hline Astigmatismo & 0,001 & 0,999 & 0,338 \\
\hline Coma & 0,061 & 0,999 & 0,082 \\
\hline Aberração esférica & 0,394 & 0,999 & 0,806 \\
\hline $\begin{array}{l}\text { Outras aberrações } \\
\text { de alta ordem }\end{array}$ & 0,003 & 0,999 & 0,025 \\
\hline Aberração total (RMS) & 0,001 & 0,999 & 0,654 \\
\hline AAO (RMS) & 0,025 & 0,999 & 0,019 \\
\hline
\end{tabular}

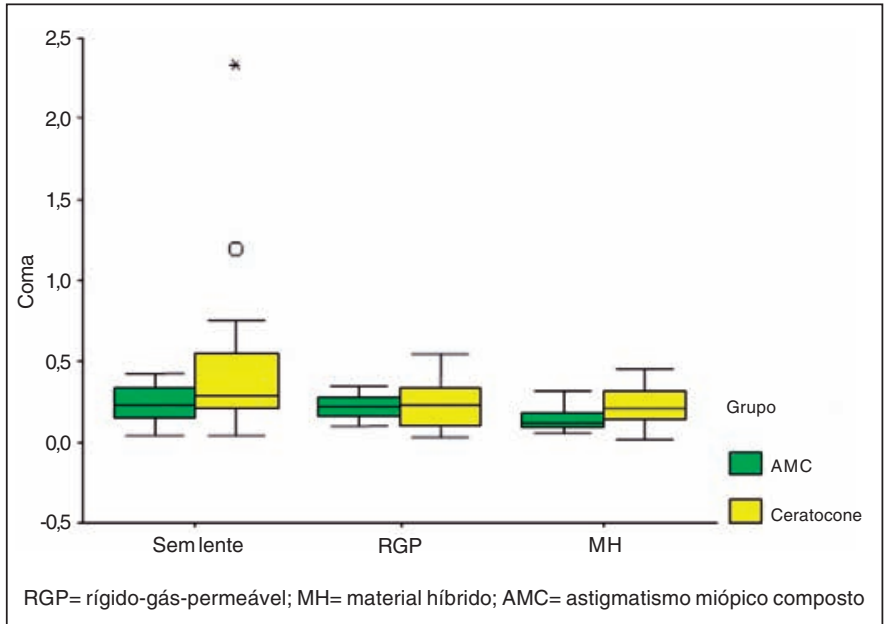

Figura 4 - Distribuição da variável coma segundo o tipo de lente de contato e o grupo de pacientes

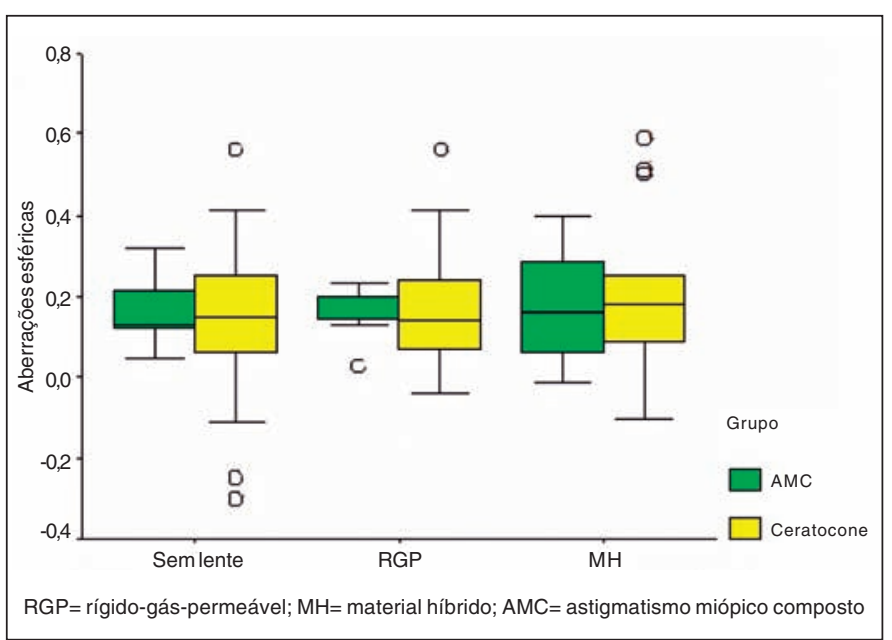

Figura 5 - Distribuição da variável aberração esférica segundo o tipo de lente de contato e o grupo de pacientes

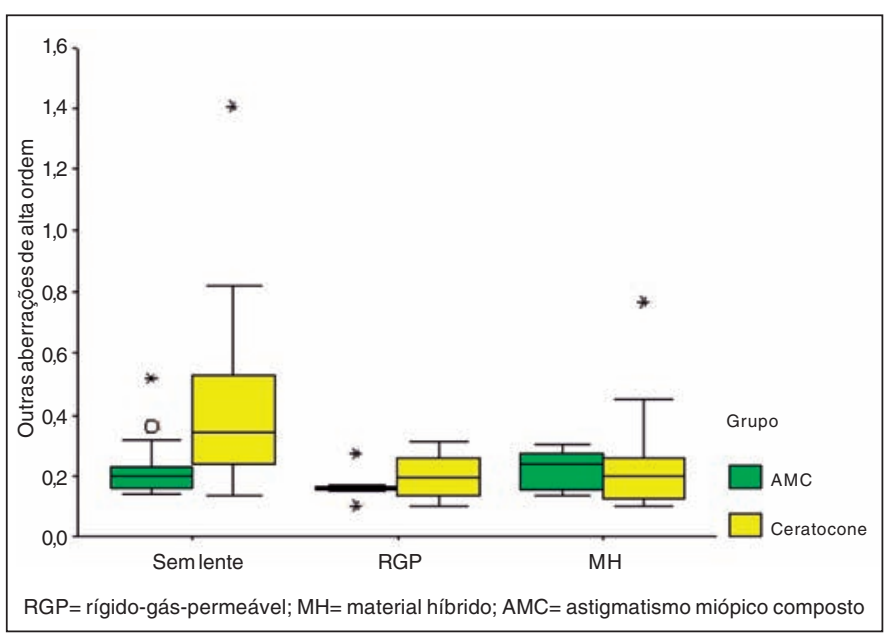

Figura 6 - Distribuição da variável outras aberrações de alta ordem segundo o tipo de lente de contato e o grupo de pacientes 


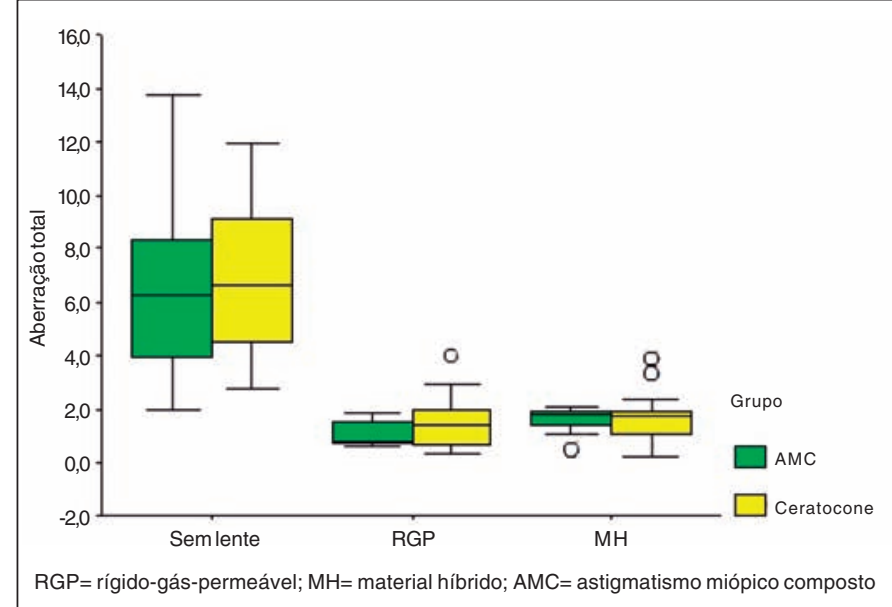

Figura 7 - Distribuição da variável aberração total segundo o tipo de lente de contato e o grupo de pacientes

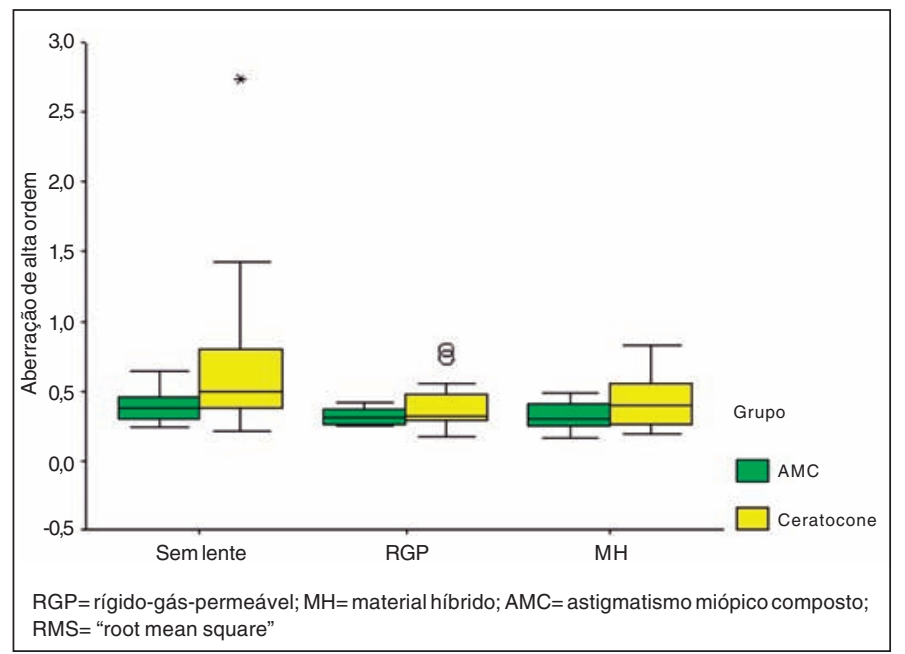

Figura 8 - Distribuição da variável aberrações de alta ordem (RMS) segundo o tipo de lente e o grupo de pacientes

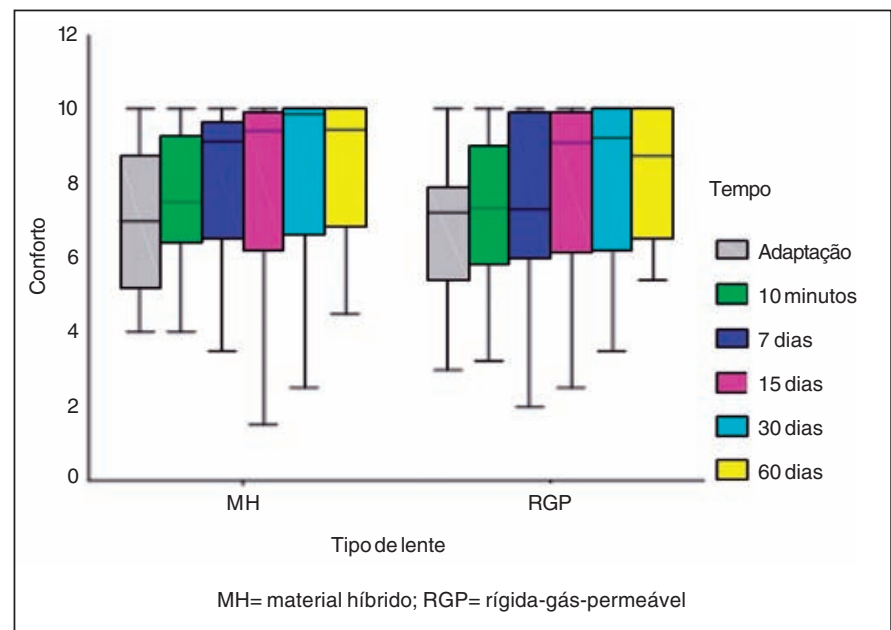

Figura 9 - Distribuição da avaliação de conforto segundo o tipo de lente de contato e o tempo em pacientes com astigmatismo miópico composto po com relação ao tempo de ruptura do filme lacrimal (BUT) ( $\mathrm{p}=0,989)$. Notou-se, no entanto, que houve diminuição do BUT $(\mathrm{p}=0,001)$ quando foram comparadas medidas antes e dois meses após o uso de lentes de contato, para ambas as lentes. Tal comportamento foi uniforme nos grupos de pacientes com ceratocone e nos pacientes com astigmatismo miópico composto $(\mathrm{p}=0,775)$.

\section{DISCUSSÃO}

Segundo a literatura, pacientes usuários de lentes RGP costumam no início queixar-se de desconforto. Uma vez que estejam bem adaptados ao uso das lentes, não há diferença significante na freqüência de dor ocular entre usuários de LC gelatinosas e lentes rígidas ${ }^{(9-10)}$. Neste estudo, o conforto medido através da escala visual analógica (EVA) não foi diferente entre os pacientes portadores de astigmatismo miópico composto e os pacientes portadores de ceratocone, como também, entre os pacientes usuários de lentes de $\mathrm{MH}$ e lentes RGP. Porém, para todos os grupos houve variação no conforto ao longo do tempo. Este aumentou até o $7^{\circ}$ dia e, então, estabilizou-se.

A EVA é um dos métodos mais aceitos de medida de dor, sendo um método simples e reprodutível para a medida de respostas subjetivas ${ }^{(10-12)}$. A mesma já foi usada em pesquisas oculares tais como: dor ocular em pacientes com uveíte ${ }^{(13)}$, sensação provocada por estimulação corneana ${ }^{(14)}$, conforto ocular durante a relação de trabalhos em terminais de vídeo ${ }^{(15)}$ e em usuários de lentes de contato rígidas ${ }^{(10)}$.

Segundo a literatura, o desconforto é a principal razão pela qual os pacientes abandonam o uso de lentes de contato ${ }^{(16-19)}$. Grande parte dos recentes avanços nos materiais de lentes de contato, desenho e formas de uso e sistemas de limpeza e manutenção das mesmas tem como objetivo reduzir o descon-

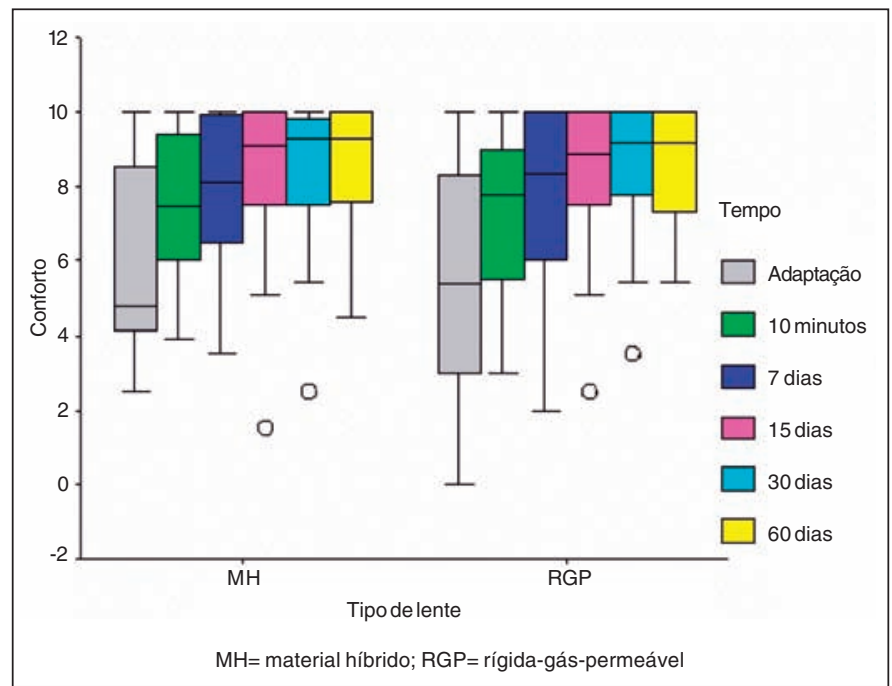

Figura 10 - Distribuição da avaliação de conforto segundo o tipo de lente de contato e o tempo em pacientes com ceratocone 
forto apresentado pelos pacientes ${ }^{(16)}$. $\mathrm{O}$ aumento do conforto e a diminuição de sintomas associados ao olho seco é apontado como o principal fator que pode evitar o descontinuamento do uso de lentes ${ }^{(20)}$.

Com relação a acuidade visual medida através da escala de Bailey e Lovie ${ }^{(21-22)}$ não houve diferença significante entre os pacientes portadores de ceratocone e os portadores com astigmatismo miópico composto, como também, entre os pacientes usuários dos dois tipos de lentes. A tabela utilizada possui como vantagens a constante progressão geométrica entre as diferentes linhas da mesma, o mesmo número de optotipos em cada linha além do mesmo grau de dificuldade na leitura destes.

Muitos estudos têm como objetivo avaliar a acuidade visual obtida com diferentes correções dos erros refrativos, mas os resultados destes não são concordantes ${ }^{(23-27)}$. A enorme variabilidade encontrada nestes estudos pode ser devida a uma constante modificação nos desenhos das lentes de contato, diferentes métodos de medida da acuidade visual e/ou amostras de pequeno tamanho ${ }^{(28)}$.

Com relação ao tempo de ruptura do filme lacrimal, observou-se redução significante quando se comparou as medidas iniciais e as medidas com dois meses de uso das lentes de contato. Inúmeros trabalhos relatam que uma redução da camada lipídica do filme lacrimal e um aumento na evaporação do filme lacrimal como um todo o que explica a redução do tempo de ruptura do filme lacrimal em usuários de lentes de contato ${ }^{(29-30)}$. Investigações relacionam a quebra do filme lacrimal com diminuição da acuidade visual e sensibilidade ao contraste ${ }^{(31-32)}$.

A instabilidade do filme lacrimal, em pacientes usuários de lentes de contato é aparentemente contraditória com o aumento da tolerância ao uso de lentes, que muitos pacientes apresentam com o decorrer do tempo, sendo que tal fato ocorreu neste estudo: enquanto houve uma redução significante, quando comparadas as medidas antes do uso com sessenta dias de uso das lentes, a medida do conforto apresentou aumento no mesmo período.

Um dos motivos que pode explicar tal situação é que o uso de lentes de contato está relacionado a uma redução da sensibilidade corneana demonstrada por inúmeros trabalhos ${ }^{(33-36)}$.

Utilizando diferentes técnicas, a medida de sensibilidade ao contraste tem sido utilizado nas pesquisas médicas na verificação do desempenho visual em usuários de lentes de contato. De um modo geral, se aceita que a sensibilidade ao contraste é superior à acuidade visual na determinação do desempenho visual em usuários de $\mathrm{LC}^{(37)}$.

Neste estudo, quando comparados os níveis de sensibilidade com o uso de lentes e óculos para o mesmo grupo de pacientes, não houve diferença significante exceto para a freqüência $\mathrm{B}$, maior para os pacientes usuários de óculos. Os resultados da literatura são divergentes ${ }^{(38-39)}$.

Com relação à análise das aberrações de alta ordem houve melhora significante em todas as aberrações avaliadas com exceção da aberração esférica e do coma que apresentaram, respectivamente, aumento e diminuição de seus valores, porém sem significância estatística. Tal fato ocorreu nos grupos de pacientes usuários de lentes RGP e MH. Estudos têm demonstrado que uso de lentes de contato está relacionado a ocorrências de aberrações, especialmente aberração esférica ${ }^{(38-40)}$. Outros apontam que o uso de lentes de contato está relacionado com a diminuição do total das aberrações oculares ${ }^{(42-44)}$.

\section{CONCLUSÃO}

As lentes de contato de material híbrido, utilizadas por portadores de ceratocone e astigmatismo miópico composto, propiciam desempenho visual e conforto satisfatórios, em níveis que não diferem, quando comparadas com a lente de contato rígida-gás-permeável nos dois grupos de pacientes.

\section{ABSTRACT}

Purpose: To evaluate comfort and visual performance in relation to two different used contact lens types: hybrid material (HM) and rigid-gas-permeable (RGP), in patients with regular myopic astigmatism and with keratoconus. Methods: A randomized, double masked, prospective study of 22 patients with the diagnosis of myopic astigmatism (8 with myopic astigmatism and 14 with keratoconus) was conducted. Fifteen patients were female and 7 were male, and mean age was: $32.13 \pm 8.12$ years. In one of the eyes a rigid-gas-permeable contact lens was adapted (DK 30), and in the other a hybrid material contact lens was adapted (DK 23). All patients were submitted to the following tests: measurement of comfort level by means of the analogical visual scale, tear break-up time, best corrected visual acuity with the Bailey-Lovie scale adapted for 4 meters, functional acuity contrast test (FACT) and wavefront analysis. Results: In relation to comfort, there was no association with the evaluated contact lens type $(\mathrm{p}=0.350)$. There was a variation in comfort level during the first 7 days. The visual acuity increased between the $7^{\text {th }}$ and the $15^{\text {th }}$ day of adaptation. Visual acuity stabilized right after this period. The visual acuity did not show differences in relation to the studied lens type. It was verified that there was no difference in the tear break-up time $(\mathrm{p}=0.989)$ in relation to the studied lenses type and there was a decrease in the tear break-up time $(\mathrm{p}=0.001)$ when we compared the measurement before the use of the lenses and 2 months after their use. There was no difference in contrast sensitivity in the used lens type and between each type and the use of glasses $(\mathrm{p}<0.047)$. High order aberrations decreased significantly when comparing patients with and without contact lenses, except for spherical and coma aberrations ( $\mathrm{p}=0.394)$. Conclusion: Comfort and visual performance when comparing hybrid material and rigid-gas-permeable contact lenses did not show any significant difference in the patients or when compared separately in regular myopic astigmatism and in keratoconus patients.

Keywords: Contact lenses; Keratoconus; Myopia; Astigmatism; Contrast sensitivity 


\section{REFERÊNCIAS}

1. Ruben M, Guillon M. Introduction. In: Ruben M, Guillon M, editors. Contact lens practice. 3rd ed. London: Chapman \& Hall Medical; 1994. p.1-2.

2. Sabell AG. Origins of the contact lens. In: Ruben M, Guillon M, editors. Contact lens practice. 3rd ed. London: Chapman \& Hall Medical; 1994. p.3-21.

3. Munoa Roiz JL, Aramendía Salvador E. Historia y desarrollo de las lentes de contacto. In: Duran de la Colina JA. Complicaciones de las lentes de contacto [monografia na Internet]. Madrid: Oftalmored; 1998 [citado 2004 Dez 10]. Disponible en: http://www.oftalmored.com/lentes/.

4. Refojo MF. Chemical composition and properties. In: Ruben M, Guillon M, editors. Contact lens practice. London: Chapman \& Hall Medical; 1994. p.22-42.

5. Rubinstein MP, Sud S. The use of hybrid lenses in management of the irregular cornea. Cont Lens Anterior Eye. 1999;22(3):87-90.

6. Contamac. Hybrid FS (Hybufocon A): product information [text on the Internet] United Kingdom: Contamac; 2005 [cited 2005 Apr 18]. Available from: http:// www.contamac.com/db/namproducts/HYBRID\%20FS\%20(Hybufocon\% 20A).

7. Neter J, Kutner M, Nachtsheim C, Wassermann W. Applied linear statistical models. 4th ed. Boston; Irwin; 1996.

8. Naeser K, Hjortdal J. Multivariate analysis of refractive data: mathematics and statistics of spherocylinders. J Cataract Refract Surg 2001;27(1):129-42. Comment in: J Cataract Refract Surg. 2002;28(2):206-7.

9. Vajdic C, Holden BA, Sweeney DF, Cornish RM. The frequency of ocular symptoms during spectacle and daily soft and rigid contact lens wear. Optom Vis Sci. 1999;76(10):705-11.

10. Fujita H, Sano K, Sasaki S, Ohno-Matsui K, Tanaka Ti, Baba T, et al. Ocular disconfort at the initial wearing of rigid gas permeable contact lenses. Jpn J Opthalmol. 2004;48(4):376-79.

11. Scott J, Huskisson EC. Graphic representation of pain. Pain. 1976;2(2):175-84

12. Sriwatanaku K, Kelvie W, Lasagna L. The quantification of pain: an analysis of works used to describe pain an analgesia in clinical trials. Clin Pharmacol Ther. 1982;32(2):143-8.

13. Zaczek A, Zetterston $C$. The effect of phenylephrine on pain and flare intensity in eyes with uveitis. Acta Ophthalmol Scand. 200;78(5):516-8.

14. Acosta MC, Tan ME, Belmonte C, Gallar J. Sensations evoked by mechanical, chemical, and thermal stimulation of the conjunctiva and cornea. Invest Ophthalmol Vis Sci. 2001;42(9):2063-7.

15. Acosta MC, Gallar J, Belmonte C. The influence of eye solutions on blinking and ocular comfort at rest and during at video display terminal. Exp Eye Res. 1999;68(6):663-9.

16. Young G, Veys J, Pritchard N, Coleman S. A multi-centre study of lapsed contact lens wearers. Ophthalmic Physiol Opt. 2002;22(6):516-27.

17. Weed KH, Fonn D, Potvin R. Discontinuation of contact lens wear. Optom. Vis Sci. 1993;70:140.

18. Briggs ST. Profile of contact lens failures in Saudi Arabia. Clin Exp Optometry. 1996;79:255-9.

19. Schlanger JL. A study of contact lens failures. J Am Optom Assoc. 1993;64 (3):220-4.

20. Pritchard N, Foonn D, Brazeau D. Discontinuation of contact lens wear: a survey. J Am Optom Assoc. 1993;64:220-4.

21. Bailey IL, Lovie JE. New design principles for visual acuity letter charts. Am J Optom Physiol Opt. 1976;53(11):740-5.
22. Ferris FL 3rd, Kassoff A, Bresnick GH, Bailey I. New visual acuity charts for clinical research. Am J Ophthalmol. 19821;94(1):91-6.

23. Applegate RA, Hilmantel G, Howland HC, Tu EY, Starck T, Zayac EJ. Corneal first surface optical aberrations and visual performance. J Refract Surg. 2000;16(5):507-14

24. Guillon M, Schock SE. Soft contact lens visual performance multicenter study. Optom Vis Sci. 1991;68(2):96-103.

25. Lohmann CP, Fitzke F, O'Brart D, Muir MK, Timberlake G, Marshall J. Corneal light scattering and visual performance in myopic individuals with spectacles, contact lenses, or excimer laser photorefractive keratectomy. Am J Ophthalmol. 1993;115(4):444-53.

26. Wachler BS, Phillips CL, Schanzlin DJ, Krueger RR. Comparison of contrast sensitivity in different soft contact lenses and spectacles. CLAO J 1999;25(1): 48-51.

27. Wechsler S. Visual acuity in hard and soft contact lens wearers: a comparison. J Am Optom Assoc. 1978;49(3):251-6.

28. Bailey MD, Walline JJ, Mitchell GL, Zadnik K. Visual acuity in contact lens wearers. Optom Vis Sci. 2001;78(10):726-31.

29. Ridder WH, 3rd, Tomlinson A. The effect of artificial tears on visual performance in normal subjects wearing contact lenses. Optom Vis Sci. 2003;80(12): 826-31.

30. Tomlinson A, Cedarstaff TH. Tear evaporation from the human eye: the effects of contact lens wear. J Br Contact Lens Assoc. 1982;5:141-50.

31. Timberlake GT, Doane MG, Bertera JH. Short-term, low-contrast visual acuity reduction associated with in vivo contact lens drying. Optom Vis Sci. 1992;69 (10):755-60.

32. Mackie IA. Contact lenses in dry eyes. Trans Ophthalmol Soc UK. 1985;104 (Pt 4): 477-83.

33. Polse KA. Etiology of corneal sensitivity changes accompanying contact lens wear. Invest Ophthalmol Vis Sci. 1978;17(12):1202-6.

34. Millodot M. Aestesiometry. In: Ruben M, Guillon M, editors. Contact lens practice. 3rd ed. London: Chapman \& Hall Medical; 1994. p.1-21.

35. Bergenske PD, Polse KA. The effect of rigid gas permeable lenses on corneal sensitivity. J Am Optom Assoc. 1987;58(3):212-5

36. Millodot M. Corneal sensitivity. Int Ophthalmol Clin. 1981;21(2):47-54.

37. Guillon M. Contact lens visual performance. In: Ruben M, Guillon M, editors Contact lens practice. 3rd ed. London: Chapman \& Hall Medical; 1994. p.951-67.

38. Millodot M. Variation of visual acuity with contact lenses. A function of luminance. Arch Ophthalmol. 1969;82(4):461-5.

39. Guillon M, Lydon DPM, Wilson C. Variations in contrast sensitivity function with spectacles and contact lenses. J Br Contact lens Assoc. 1983;60(3):120-4.

40. Dorronsoro C, Barbero S, Llorente L, Marcos S. On-eye measurement of optical performance of rigid gas permeable contact lenses based on ocular and corneal aberrometry. Optom Vis Sci. 2003;80(2):115-25.

41. Hong X, Himebaugh N, Thibos LN. On-eye evaluation of optical performance of rigid and soft contact lenses. Optom Vis Sci. 2001;78(12):872-80.

42. Westeheimer G. Aberrations of contact lenses. Am J Optom Arch Am Acad Optom. 196;38:445-8.

43. Cox I. Theorical calculation of the longitudinal spherical aberration of rigid and soft contact lenses. Optom Vis Sci. 1990;67(4):277-82.

44. Hammer RM, Holden BA. Spherical aberration of aspheric contact lenses on eye. Optom Vis Sci. 1994;71(8):522-8

\section{Nos artigos enviados para publicação, o nome dos autores} e suas afiliações devem estar completos. Isso facilitará a indexação e os links com as bases de dados e o CV Lates. 